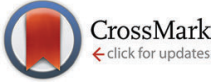

Cite this: Phys. Chem. Chem. Phys., 2016, 18, 5753

Received 15th August 2015,

Accepted 21st September 2015

DOI: $10.1039 / \mathrm{c} 5$ cp04858c

www.rsc.org/pccp

\section{Detection of correlated conformational fluctuations in intrinsically disordered proteins through paramagnetic relaxation interference $\dagger$}

\author{
D. Kurzbach, $\ddagger^{a}$ A. Vanas, $\ddagger^{a}$ A. G. Flamm, $\ddagger^{a}$ N. Tarnoczi, ${ }^{a}$ G. Kontaxis, ${ }^{a}$ \\ N. Maltar-Strmečki, ${ }^{b}$ K. Widder, ${ }^{b}$ D. Hinderberger ${ }^{b}$ and R. Konrat*a
}

Functionally relevant conformational states of intrinsically disordered proteins (IDPs) are typically concealed in a vast space of fast interconverting structures. Here we present a novel methodology, NMR-based paramagnetic relaxation interference (PRI), that allows for direct observation of concerted motions and cooperatively folded sub-states in IDPs. The proposed NMR technique is based on the exploitation of cross correlated electron-nuclear dipolar relaxation interferences in doubly spin-labeled proteins and probes the transient spatial encounter of electron-nucleus spin pairs.

\section{Introduction}

Intrinsically disordered proteins (IDPs) constitute important hubs in protein interaction networks. ${ }^{1}$ They play key roles in cellular regulatory linkages thus aiding to maintain the physiological equilibrium. A malfunction is frequently accompanied by severe malady like cancer or neurodegenerative diseases. ${ }^{2,3}$ However, to date IDP function and structure-function relations have been only vaguely understood. ${ }^{4,5}$ Although these highly dynamic proteins sample vast and heterogeneous conformational spaces there is evidence for short-lived preformation of binding sites in otherwise disordered structural ensembles. ${ }^{6,7}$ Paramagnetic relaxation enhancement (PRE) has been established as a powerful tool for probing these sparsely populated and conformationally dynamic states. A paramagnetic spin label is used to enhance the transverse relaxation rates, $R_{2}$, of amide protons $\left({ }^{1} \mathrm{H}^{\mathrm{N}}\right)$ in the protein backbone by a rate ${ }^{1} \mathrm{H}^{\mathrm{N}}-\Gamma_{2}$. The magnitude of ${ }^{1} \mathrm{H}^{\mathrm{N}}-\Gamma_{2}$ is dependent on the correlation function of the dipole between $\mathrm{H}^{\mathrm{N}}$ and the unpaired electron, $\mathrm{X}$. It decays with a correlation time $\tau_{\mathrm{c}}$ and the average distance between $\mathrm{H}^{\mathrm{N}}$ and $\mathrm{X}$ by $\left\langle r_{\mathrm{HN}-\mathrm{X}}{ }^{-6}\right\rangle .^{8-10} \tau_{\mathrm{c}}$ denotes the effective reorientation time of the amide-electron $\left(\mathrm{H}^{\mathrm{N}}-\mathrm{X}\right)$ vector. ${ }^{11,12}$ For two-spin $\mathrm{H}^{\mathrm{N}}-\mathrm{X}$ systems ${ }^{1} \mathrm{H}^{\mathrm{N}}-\Gamma_{2}$ quantifies the PRE as ${ }^{1} \mathrm{H}^{\mathrm{N}}-\Gamma_{2}=$ $(1 / 15)\left((h / 2 \pi) \mu_{0} \gamma_{\mathrm{H}} g \mu_{\mathrm{B}} / 4 \pi\right)^{2} S(S+1)\left[4 J(0)+3 J\left(\omega_{\mathrm{L}}\right)\right]\left\langle r_{\mathrm{HN}-\mathrm{X}}{ }^{-6}\right\rangle$, where all symbols have their usual meaning. ${ }^{8}$ Yet, the quantification

\footnotetext{
${ }^{a}$ Department for Structural and Computational Biology Max F. Perutz Laboratories, University of Vienna Vienna Biocenter Campus 5, 1030 Vienna, Austria. E-mail: Robert.Konrat@univie.ac.at

${ }^{b}$ Institute for Physical Chemistry Martin-Luther-Universität Halle-Wittenberg von-Danckelmann-Platz 4, 06120 Halle (Saale), Germany

$\dagger$ Electronic supplementary information (ESI) available. See DOI: $10.1039 / \mathrm{c} 5 \mathrm{cp} 04858 \mathrm{c}$

\$ These authors contributed equally to this work.
}

of PREs, $\tau_{c}$ and the spectral density functions $J(\omega)$ is not straightforward for IDPs. ${ }^{13}$

Several applications of IDPs have been reported demonstrating the validity of the PRE approach. ${ }^{8,14,15}$ However, since all thermally accessible sub-states contribute to the observed PRE the existence of concerted motions and cooperatively folded segments could not unambiguously be detected. To understand correlated (concerted) structural fluctuations in IDPs we propose a novel technique that exploits paramagnetic relaxation interference (PRI) effects. In our approach and in contrast to conventional PRE techniques we exploit dipole-dipole crosscorrelation effects between two spin labels carrying electrons $\mathrm{X}(1)$ and $\mathrm{X}(2)$ and an amide proton ${ }^{1} \mathrm{H}^{\mathrm{N}}$.

The novel PRI methodology involves PRE measurements using single spin labels, $\mathrm{X}(1)$ and $\mathrm{X}(2)$, to determine the rates ${ }^{1} \mathrm{H}^{\mathrm{N}}-\Gamma_{2}[\mathrm{X}(1)]$ and ${ }^{1} \mathrm{H}^{\mathrm{N}}-\Gamma_{2}[\mathrm{X}(2)]$, and a double mutant containing spin labels at both residual positions, $\mathrm{X}(1)$ and $\mathrm{X}(2)$, to determine ${ }^{1} \mathrm{H}^{\mathrm{N}}-\Gamma_{2}[\mathrm{X}(1)+\mathrm{X}(2)]$. The PRI effect is quantified as $\Delta \Delta^{1} \mathrm{H}^{\mathrm{N}}-\Gamma_{2}={ }^{1} \mathrm{H}^{\mathrm{N}}-\Gamma_{2}[\mathrm{X}(1)+\mathrm{X}(2)]-\left\{{ }^{1} \mathrm{H}^{\mathrm{N}}-\Gamma_{2}[\mathrm{X}(1)]+{ }^{1} \mathrm{H}^{\mathrm{N}}-\Gamma_{2}[\mathrm{X}(2)]\right\}$. The simultaneous presence of two unpaired electrons in spatial proximity leads to interference effects between the $\mathrm{X}(1)-{ }^{1} \mathrm{H}^{\mathrm{N}}$ and $\mathrm{X}(2)-{ }^{1} \mathrm{H}^{\mathrm{N}}$ dipoles. Analogous cross-correlated relaxation mechanisms have been utilized to great advantage in TROSY and Methyl-TROSY techniques to study high-molecular weight systems. ${ }^{16,17}$ Beyond that cross-correlated NMR relaxation has attracted substantial interest in the past as a powerful tool for studying structure and dynamics of proteins in solution. ${ }^{18}$ Cross-correlated relaxation arises from interference effects between the fluctuations of two different relaxation mechanisms of the same rank, which are active simultaneously and in a correlated manner. These concerted effects have been shown to be a valuable source of information about the structure and 
dynamics of proteins, since they are dependent on the relative geometry of the spin system. Typically cross-correlated interference effects can be observed between two different dipolar (D) interactions (D-D), two different chemical shift anisotropy (CSA-CSA) tensors or between a dipolar and a chemical shift anisotropy (D-CSA) interaction. Here a novel $\mathrm{X}(1)-{ }^{1} \mathrm{H}^{\mathrm{N}}$ and $\mathrm{X}(2)-{ }^{1} \mathrm{H}^{\mathrm{N}}$ electron-nucleus dipole-dipole interference term is exploited.

\section{Results and discussion}

The principle of the presented technique is outlined in Fig. 1. For illustration, we consider the hypothetical case of an IDP that exists in several conformations (I, II and III) displaying differing local compaction of the polypeptide chain. In conformations I and II either the $\mathrm{N}$ - or the C-terminal segment is folded and long-range PRE effects are therefore separately observed only for residues located in the compacted regions. The two structured segments undergo uncorrelated foldingunfolding transitions. Thus, the observed PRE effect in the doubly labeled sample is equal to the sum of the individual single-electron (PRE) contributions. In contrast, if conformation III is significantly populated in the ensemble experimental PREs observed for the doubly labeled samples are altered due to dipole-dipole cross-correlation/interference effects. In the doubly labeled case (right side of Fig. 1) significant deviations from the additive sum, ${ }^{1} \mathrm{H}^{\mathrm{N}}-\Gamma_{2}[\mathrm{X}(1)]+{ }^{1} \mathrm{H}^{\mathrm{N}}-\Gamma_{2}[\mathrm{X}(2)]\left(\Delta^{1} \mathrm{H}^{\mathrm{N}}-\Gamma_{2} \neq 0\right)$, are thus observed. The magnitude of $\Delta^{1} \mathrm{H}^{\mathrm{N}}-\Gamma_{2}$ is primarily dependent on a term $\left\langle 3 \cos ^{2} \theta-1\right\rangle$, where $\theta$ is the angle between the $\mathrm{X}(1)-{ }^{1} \mathrm{H}^{\mathrm{N}}$ and $\mathrm{X}(2)-{ }^{1} \mathrm{H}^{\mathrm{N}}$ dipoles. The angle brackets indicate the effective (" $r_{\mathrm{HN}-\mathrm{X}}{ }^{-6}$ filtered") ensemble (and time) average. We want to emphasize that PRI depicts exclusively properties of the compacted state of a structurally dynamic protein, since the $\left\langle r_{\mathrm{HN}-\mathrm{X}}{ }^{-6}\right\rangle$ dependence entails a steep distance dependence of the underlying PRE. Given that individual PRI-effects can be measured as a function of residue and spin label positions this
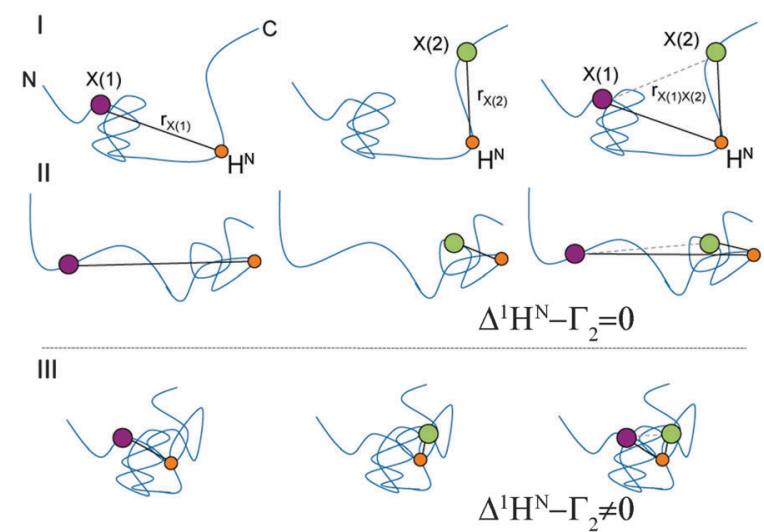

Fig. 1 Schematic representation of segmentally folded (I and II) and entirely compacted (III) conformations of an IDP. Purple and green dots indicate spin-labeling sites, $X(1)$ and $X(2)$, orange dots an amide, $\mathrm{H}^{\mathrm{N}}$, of interest. In the compact state the distance $r_{\mathrm{X}(1) \times(2)}$ shortens and thus leading to electron nucleus dipolar interferences. ${ }^{1} \mathrm{H}^{\mathrm{N}}-\Gamma_{2}$ rates. novel technique allows for direct experimental assessment of correlated (concerted) structural fluctuations in IDPs.

We illustrate this novel methodology with an application to the IDP Osteopontin (OPN) and the brain acid soluble protein 1 (BASP1). ${ }^{19}$ It is well-known that OPN samples are extended as well as small populations of transiently folded and compacted conformations ( $c f$. Fig. 1). ${ }^{20}$ The latter are central to substrate interactions. ${ }^{14,21}$ For OPN we performed PRI measurements on a 220 amino acid (aa) long metastasis-associated, ${ }^{21}$ truncated form (aa 47-264 of full-length OPN). Four singly and six doubly spin-labelled OPN cysteine mutants were produced by combining four labelling sites: C54, C108, C188 and C247. ${ }^{20}$ MTSL ( $S$-(1-oxyl-2,2,5,5-tetramethyl-2,5-dihydro- $1 H$-pyrrol-3-yl)methyl methanesulfonothioate) labels were used to introduce stable unpaired electrons. Fig. 2a-c, display prototypical $P R E{ }^{1} \mathrm{H}^{\mathrm{N}}-\Gamma_{2}$ data obtained for residues 68 of C108-C188, 119 of C108-C18 and 226 of $\mathrm{C} 54-247 .{ }^{1} \mathrm{H}^{\mathrm{N}}-\Gamma_{2}$ rates were obtained using threepoint measurements as adapted from the approach of Clore and co-workers. ${ }^{22}$ We observed significantly different PRIs, $\Delta^{1} \mathrm{H}^{\mathrm{N}}-\Gamma_{2}$, between the different residues indicating significant heterogeneity of the conformational ensemble of OPN in solution. In order to rule out intermolecular PRE effects control experiments were performed using ${ }^{14} \mathrm{~N}$-MTS-labeled and ${ }^{15} \mathrm{~N}$ wild-type (for detection) OPN. Corresponding data are given in the ESI. $\dagger$ To demonstrate that indeed electron-nucleus dipoledipole interference terms constitute the relevant factors we measured PRE data at different fields (600 and $800 \mathrm{MHz}$ ). The obtained values were found to be independent of magnetic field strength thus corroborating the relevance of dipoledipole cross-correlation effects (see ESI $\dagger$ ). The latter is further validated by electron $T_{1}$ times that have been exemplarily determined for the system C54-C188 at $50 \mathrm{~K}$ : C54: $804 \pm 4 \mu \mathrm{s}$,
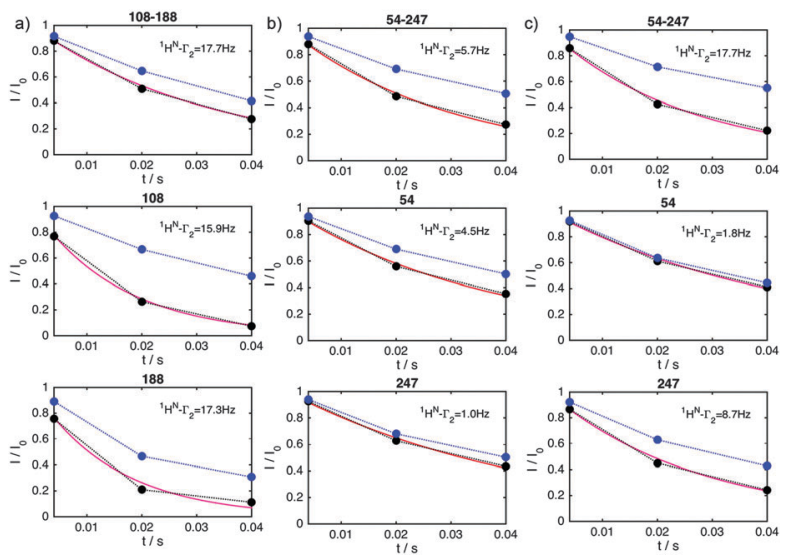

Fig. 2 Prototypical ${ }^{1} \mathrm{H}^{\mathrm{N}}-\mathrm{R}_{2}$ relaxation measurements for residues displaying negative (a), vanishing (b) or positive (c) dipole-dipole crosscorrelation effects. ${ }^{1} \mathrm{H}^{\mathrm{N}}$ data are shown for residues 125 of $\mathrm{C} 108-\mathrm{C} 188$ (a), 115 of C54-C247 (b) and 227 of C54-C247 (c) in doubly spin labeled (top) and the corresponding singly labeled OPN mutants (black: active MTSL, blue: reduced MTSL). The obtained ${ }^{1} \mathrm{H}^{\mathrm{N}}-\Gamma_{2}$ relaxation rates are also given and were extracted by fitting the data to $I / I_{0}=\exp \left(-R_{2} t\right)$ (not shown). The obtained rates were in agreement with a model for transverse relaxation in AMX spin systems developed by Kay and Bull (purple fits, see the text). 
C188: $710 \pm 11 \mu$ s and C54-C188: $706 \pm 5 \mu$ s. These values indicate the spatial proximity of the two spin labels in the double mutant allowing for significant inter-electron dipolar coupling. Finally, good agreement between experimental and theoretical ${ }^{1} \mathrm{H}^{\mathrm{N}}-\Gamma_{2}$ rates was obtained employing a formalism described by Kay and co-workers ${ }^{23}$ for transverse relaxation in AMX spin systems (see Fig. 2). During the relaxation period non-vanishing dipole-dipole CCRs lead to mixtures of in-phase and doubly anti-phase coherences and thus to differential weighting of the individual multiplet components $(\alpha \alpha, \beta \alpha, \alpha \beta$ and $\beta \beta)$, depending on the sign of CCR. Most importantly, and as shown by Kay and co-workers ${ }^{23}$ the diagonal elements of the (transverse) relaxation matrix $(\mathrm{R}(\alpha \alpha, \alpha \alpha)=\mathrm{R}(\beta \beta, \beta \beta)$ and $\mathrm{R}(\alpha \beta, \alpha \beta)=\mathrm{R}(\beta \alpha, \beta \alpha))$ are larger than the off-diagonal (cross-relaxation) elements (e.g., $\mathrm{R}(\alpha \alpha, \alpha \beta))$. The only sizeable off-diagonal element is $\mathrm{R}(\alpha \beta, \beta \alpha)$, the inter-electron NOE as it is determined by the zero-frequency spectral density function $J\left(\omega_{\mathrm{eA}}-\omega_{\mathrm{eB}}\right), \omega_{\mathrm{eA}}$ and $\omega_{\mathrm{eB}}$ being the (identical) electron Larmor frequencies. Contributions from electron longitudinal relaxation $\left(T_{1}\right)$ were estimated based on experimental electron $T_{1}$ values. ${ }^{24,25}$ Taking this and published electron $T_{1}$ values into account and assuming a protein correlation time of about $10 \mathrm{~ns}$ suggests an electron $T_{1}$ for the proteinbound MTSL of several tenth of $\mu$ s for the magnetic field strength used in our study. It can thus be concluded that the inter-electron dipolar cross-relaxation $\mathrm{R}(\alpha \beta, \beta \alpha)$ plays a pivotal role and that the individual multiplet components do not uniformly interchange. Further fundamental insights can be obtained by formulating spin relaxation in the framework of singlet and triplet states in radical pairs employing product operators. In the product basis $\{\alpha, \beta\}$ the density operator for the singlet state is $S_{0}=1 / \sqrt{ } 2(\alpha \beta-\beta \alpha)$, while the triplet states are defined as $\left\{T_{1+}=1 / \sqrt{ } 2(\alpha \alpha), T_{1-}=\right.$ $1 / \sqrt{ } 2(\beta \beta)$ and $\left.T_{10}=1 / \sqrt{ } 2(\alpha \beta+\beta \alpha)\right\}$. Furthermore, the singlet state can be written as a linear combination of the identity matrix E, longitudinal two-spin order $\mathrm{S}_{\mathrm{ZA}} \mathrm{S}_{\mathrm{ZB}}$ and zero-quantum coherence $\{\mathrm{ZQC}\}_{x}, S_{0}=1 / 4 E-\mathrm{S}_{\mathrm{ZA}} \mathrm{S}_{\mathrm{ZB}}+\{\mathrm{ZQC}\}_{x}$. Likewise the triplet state $T_{10}$ can be formulated as $T_{10}=1 / 4 E-\mathrm{S}_{\mathrm{ZA}} \mathrm{S}_{\mathrm{ZB}}-$ $\{Z Q C\}_{x}$. Dipole-dipole cross-correlation leads to a mixture of in-phase $\mathrm{H}_{\mathrm{X}}$ and doubly anti-phase $4 \mathrm{H}_{\mathrm{X}} \mathrm{S}_{\mathrm{ZA}} \mathrm{S}_{\mathrm{ZB}}$, which can be written as linear combination of $\mathrm{H}_{\mathrm{X}} S_{0}$ and $\mathrm{H}_{\mathrm{X}} T_{10} \cdot{ }^{26}$ It is well established that singlet-states show favorable relaxation properties. ${ }^{27,28}$ For example, inter-electron dipolar interactions are not relevant for the relaxation of the singlet-state $S_{0}$. Chemical shift anisotropy (or g-anisotropy) contributions are reduced, too. Therefore, we conclude that the lifetime of $\mathrm{H}_{\mathrm{X}} S_{0}$ will be significant and sufficiently long to be observable in the NMR experiment. Differential relaxation due to dipole-dipole crosscorrelation will thus manifest itself in different (effective) transverse relaxation times $\Gamma_{2}{ }^{-1} \mathrm{H}^{\mathrm{N}}$.

Fig. 3 shows experimental residue plots of PRI values obtained for the six doubly labelled OPN systems (C54-C188, C54-C108, C108-C247, C108-C188, C188-C247 and C54-C247). The data clearly show that significantly different PRIs are obtained depending on the positions of the spin label(s). The observation of significant PRIs indicates the simultaneous spatial proximity of the two labelling sites in the vicinity of the observed ${ }^{1} \mathrm{H}^{\mathrm{N}}$. Thus, concerted participation of individual protein segments in the formation of a compacted state of an IDP may be deduced from the PRI-residue plots in Fig. 3.

While largely positive PRIs were observed for the doublemutants C54-C247 and C188-C247, negative values were found when the spin labels were attached in the central (core) region 100-190 of OPN. It can thus be concluded that the conformational ensemble contains significantly compacted substates with distinct spatial arrangements. In these compact states conformational averaging is limited resulting in nonzero averaging of the geometric term $\left\langle 3 \cos ^{2} \theta-1\right\rangle$. This is in agreement with our earlier findings that localized a compacted
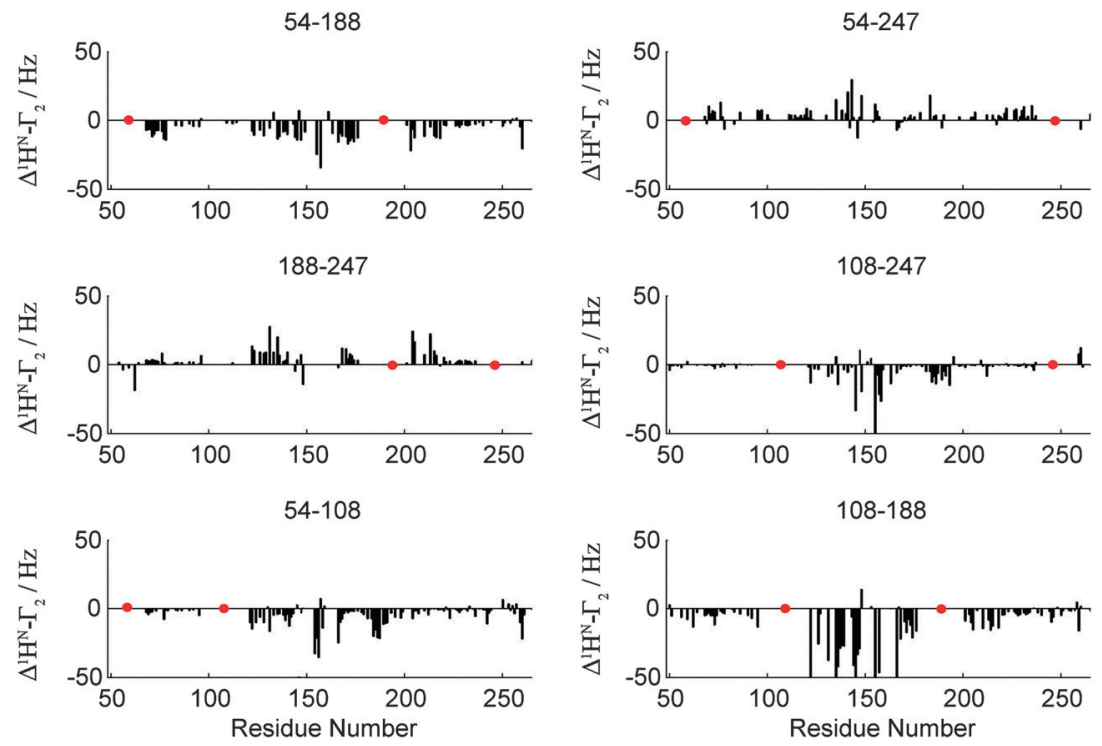

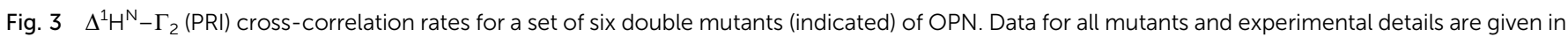
the ESI.† Red dots indicate labeling sites. 

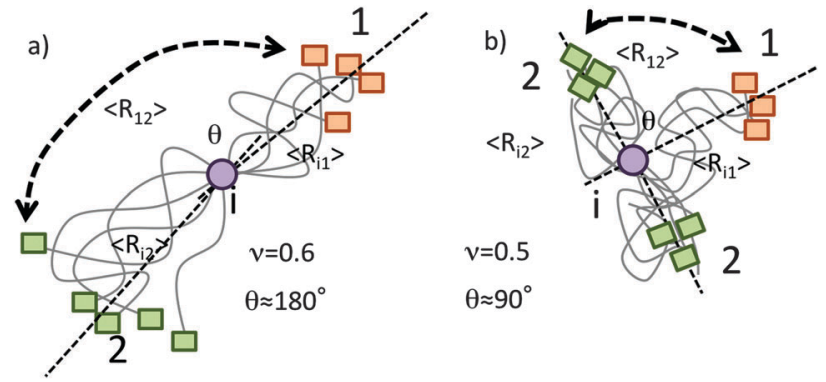

Fig. 4 Polymer theory based explanation of experimentally observed electron-nucleus dipole-dipole interference terms. Assuming real chain models the average end-to-end distance is dependent on the number of monomers in between the two ends, $\mathrm{N}$, a scaling exponent, $\nu$, and the length of the monomer units. Assuming similar exponents for the segments $X(1)-{ }^{1} H^{N}$ and $X(2)-{ }^{1} H^{N}$ it can be shown that the following relationship between projection angle $\theta$ and the exponent $\nu$ holds: $\cos \theta=1-2^{2 \nu-1}$. (a) In extended segments (chain repulsion; $\nu \approx 0.6$ ) the average projection angle $\theta$ is closer to $180^{\circ}$ thus resulting in large and positive crosscorrelation rates as $\Delta^{1} H^{N}-\Gamma_{2} \propto\left\langle 3 \cos ^{2} \theta-1\right\rangle$. (b) In a globular (ideal coil; $\nu \approx 0.5)$ state negative PRIs, $\Delta^{1} H^{N}-\Gamma_{2}<0$, are observed.

conformation to this region between aa 100 and 190 of $\mathrm{OPN}^{20,21}$

At first glance, it seems to be counterintuitive that individual segments in IDPs display significantly different conformational averaging with sizeable residue $\left\langle 3 \cos ^{2} \theta-1\right\rangle$. However, it is illustrative to analyze the dipole-dipole interference terms in the framework of Flory's theory for polymers. An outline is given in Fig. 4. Depending on the stiffness/extension of the amino acid chain (quantified by the scaling exponent, $\nu$ ) the average angle inclined by the two dipole vectors $\mathrm{X}(1)-{ }^{1} \mathrm{H}^{\mathrm{N}}$ and $\mathrm{X}(2)-{ }^{1} \mathrm{H}^{\mathrm{N}}$ can significantly vary. The mean square end-to-end distance between two residues separated by $\mathrm{N}$ amino acids can be expressed as $\left\langle r^{2}\right\rangle \propto l_{\mathrm{m}} \mathbf{N}^{\nu}\left(l_{\mathrm{m}}\right.$ being the length of the monomer unit). In extended chains (due to repulsive forces along the chain) one finds $0.5<\nu<0.6$ leading to an average projection angle $\theta$ closer to $180^{\circ}$ and thus entailing large positive PRIs since $\Delta^{1} \mathrm{H}^{\mathrm{N}}-\Gamma_{2}, \propto\left\langle 3 \cos ^{2} \theta-1\right\rangle$ holds. In contrast, in globular ideal coil or collapsed segments one finds $0.3<\nu<0.5$ and $\theta$ is closer to $90^{\circ}$. This is accompanied by negative cross-correlation rates. The observation that the compacted core segment (100190 of OPN $)^{14,20,21}$ displays negative PRI is in good agreement with our theoretical (Flory polymer theory) considerations and points towards the reliability of the proposed method.

A second example for the applicability of our PRI method was found with the human brain acid soluble protein 1 (BASP1). Earlier studies about changes in transverse relaxation times upon variations in environmental $\mathrm{pH}$ on the chicken analogue of BASP1 suggest that this protein samples $\mathrm{N}$-terminally compacted states between aa 1 and 60 of the primary sequence. ${ }^{19}$ A PRI analysis similar to that of OPN described above (see the ESI $\dagger$ for the full data set) evidently displays the N-terminal compaction of BASP1. A spin label at position C3 of the protein chain leads to strong negative PRIs for the N-terminal residues irrespective of the position of the second spin label (C92, C136, C205, see Fig. 5). Like for OPN the observed negative cross-correlation rates are in accordance with small $\theta$ values as expected for compacted polypeptide chains. For other spin label positions we observe nearly vanishing $\Delta^{1} \mathrm{H}^{\mathrm{N}}-\Gamma_{2}$. Our observations clearly indicate that the $\mathrm{N}$-terminal region of BASP1 transiently samples compact substrates despite the lack of significant secondary ${ }^{13} \mathrm{C}$ backbone chemical shifts for that regions.

\section{Conclusions}

Concluding, paramagnetic relaxation interference (PRI) exploits relaxation interference between different electron-nucleus dipoles
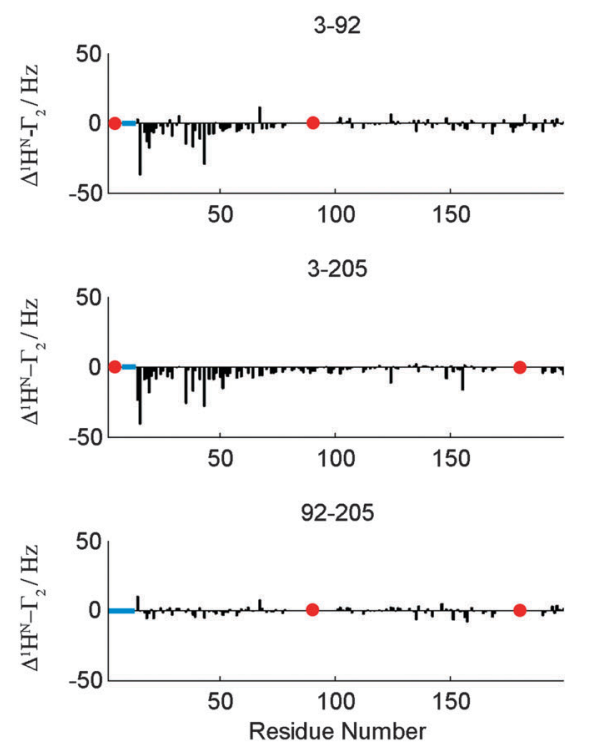
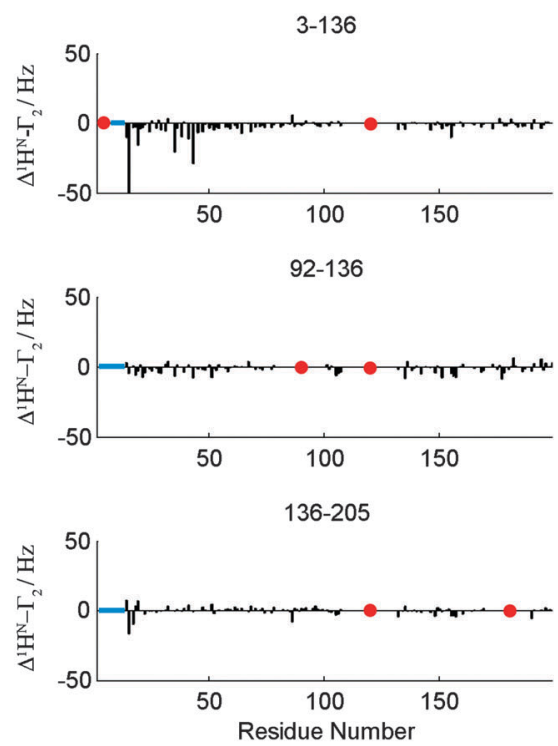

Fig. $5 \Delta^{1} H^{N}-\Gamma_{2}$ (PRI) cross-correlation rates for a set of six double mutants (indicated) of BASP1. Data for all mutants and experimental details are given in the ESI. $\uparrow$ Note that PRI data directly around the indicated labelling sites have been removed due to very low signal intensities at these positions. However, a PRI effect directly around labeling sites would be expected if signal intensities would allow for its detection. Red dots indicate labeling sites. 
in protein samples carrying two paramagnetic spin labels and yields unique insight into correlated conformation fluctuations of IDPs thereby going beyond static pictures of protein conformations. In contrast to conventional paramagnetic (PRE) NMR techniques PRI probes (transient) triple-contacts between two electrons and ${ }^{1} \mathrm{H}^{\mathrm{N}} \mathrm{S}$ of the protein backbone and reveals unique information about folded, low-populated conformations. Information about structural dynamics of IDPs and their many different conformers is important as the associated biological processes are frequently controlled by mechanisms like protein domain exchanges/swapping, conformational adaptations or switches, induced-fit and conformational selection-type events. Given its ease of implementation, we anticipate that PRI measurements will significantly broaden the realm of NMR applications in IDP research. In addition to the applications described here the unexpected example of a long-lived spin state involving radical pairs in doubly spin-labeled proteins also offers exciting possibilities for NMR structural biology of folded (globular) proteins. Experiments exploring the possibilities of (structurebased) signal assignment and structure calculation and refinement applications are currently underway in our laboratory.

\section{Experimental}

\section{Protein preparation}

The expression of recombinant natively and ${ }^{15} \mathrm{~N}$ labeled quail OPN was carried out as described previously. ${ }^{20,21}$ Human BASP1 mutants were sub-cloned into pet29b expression vectors and expressed similar to a procedure described by Geist et al. ${ }^{19}$ After purification via His-affinity chromatography His-tags were cleaved with Thrombin $\left(4{ }^{\circ} \mathrm{C}\right.$, over night). Proteins were tagged with MTSL. ${ }^{20,21}$ The labeling efficiency was always $>95 \%$ as determined via DTNB assays. Excess MTSL was removed through dialysis into PBS.

For NMR PBS containing $10 \% \mathrm{D}_{2} \mathrm{O}$ was used as a lock solvent. Concentrations were $0.7 \mathrm{mM}$ for OPN and $0.3 \mathrm{mM}$ for BASP1. For EPR the samples were concentrated to $0.7 \mathrm{mM}$.

For PRE determination the MTSL labels were reduced with ascorbic acid for referencing.

\section{NMR measurements}

NMR spectra were recorded at $25{ }^{\circ} \mathrm{C}$ on Varian spectrometers operating at 600 and $800 \mathrm{MHz}$. NMR spectra were processed with NMRPipe ${ }^{29}$ and analyzed using SPARKY. Spectra were recorded in the PFG sensitivity-enhanced mode for quadrature detection in the ${ }^{15} \mathrm{~N}$ indirect dimension with carrier frequencies for ${ }^{1} \mathrm{H}^{\mathrm{N}}$ and ${ }^{15} \mathrm{~N}$ of $4.73 \mathrm{ppm}$ and $120 \mathrm{ppm}$, respectively. A squared and $60^{\circ}$ phase-shifted sine bell window function was applied in all dimensions for apodization. Time domain data were zero-filled to twice the data set size, prior to Fourier transformation.

PRE rates of $15 \mathrm{~N}$-labeled OPN mutants C54, C108, C188, C247, C54-C108, C54-C188, C54-C247, C108-C188, C108C247, C188-C247 and of BASP mutants C3, C92, C136, C205, C3-C92, C3-C136, C3-205, C92-C136, C92-C205, C136-C205 were obtained with three-point measurements as adapted from the approach by Clore and co-workers. ${ }^{22}$

\section{EPR measurements}

All experiments were carried out using a Bruker ELEXSYS E580 spectrometer operating at $<9.4 \mathrm{GHz}$ (X-band) with a Flexline split ring resonator ER 4118X MS3. The resonator was overcoupled giving a $Q$ factor of approximately 100 . The video bandwidth was set to be $50 \mathrm{MHz}$. All measurements were made at $50 \mathrm{~K}$ with the sample in a frozen glassy state, established using a closed cycle cryostat (ARS Inc.). A two-pulse electronspin-echo (ESE) sequence $(\pi / 2-\tau-\pi-\tau$-echo) with a $\pi$ pulse width of $40 \mathrm{~ns}$ was used to detect the echo decay spectra. A-two-step phase cycle was employed to eliminate unwanted echoes. The inversion recovery technique $\left(\pi-t_{1}-\pi / 2-\tau-\pi-\right.$ echo $)$ was used to measure the longitudinal relaxation time, $T_{1}$, using an echo detection sequence with a pulse separation time of $200 \mathrm{~ns}$ and a $\pi$ pulse duration of 32 ns. $T_{1}$ time was deduced form the magnetization recovery curves fitted to the mono exponential function. Measurements of both relaxation times were performed at the field position that respond to the maximum of field-swept ESE detected spectra. The ESE detected spectra were simulated with Matlab ${ }^{\circledR}$ using the Easy-spin routines. ${ }^{30,31}$

\section{Acknowledgements}

DK thanks the Alexander von Humboldt Foundation for a Feodor-Lynen scholarship. This work was supported in part by the FWF (P26317-B21 and W-1221-B03).

\section{Notes and references}

1 H. J. Dyson and P. E. Wright, Nat. Rev. Mol. Cell Biol., 2005, 6, 197-208.

2 M. M. Babu, R. van der Lee, N. S. de Groot and J. Gsponer, Curr. Opin. Struct. Biol., 2011, 21, 432-440.

3 P. Tompa, Trends Biochem. Sci., 2012, 37, 509-516.

4 D. M. Korzhnev and L. E. Kay, Acc. Chem. Res., 2008, 41, 442-451.

5 K. Sugase, J. C. Lansing, H. J. Dyson and P. E. Wright, J. Am. Chem. Soc., 2007, 129, 13406-13407.

6 C. K. Fisher and C. M. Stultz, Curr. Opin. Struct. Biol., 2011, 21, 426-431.

7 M. R. Jensen and M. Blackledge, Proc. Natl. Acad. Sci. U. S. A., 2014, 111, E1557-E1558.

8 G. M. Clore and J. Iwahara, Chem. Rev., 2009, 109, 4108-4139.

9 N. Bloembergen and L. O. Morgan, J. Chem. Phys., 1961, 34, 842-850.

10 I. Solomon, Phys. Rev., 1955, 99, 559-565.

11 C. Charlier, S. N. Khan, T. Marquardsen, P. Pelupessy, V. Reiss, D. Sakellariou, G. Bodenhausen, F. Engelke and F. Ferrage, J. Am. Chem. Soc., 2013, 135, 18665-18672.

12 N. Bloembergen, J. Chem. Phys., 1957, 27, 572-573. 
13 A. Soranno, B. Buchli, D. Nettels, R. R. Cheng, S. MullerSpath, S. H. Pfeil, A. Hoffmann, E. A. Lipman, D. E. Makarov and B. Schuler, Proc. Natl. Acad. Sci. U. S. A., 2012, 109, 17800-17806.

14 D. Kurzbach, T. C. Schwarz, G. Platzer, S. Hofler, D. Hinderberger and R. Konrat, Angew. Chem., Int. Ed., 2014, 53, 3840-3843.

15 M. R. Jensen, R. W. H. Ruigrok and M. Blackledge, Curr. Opin. Struct. Biol., 2013, 23, 426-435.

16 J. E. Ollerenshaw, V. Tugarinov and L. E. Kay, Magn. Reson. Chem., 2003, 41, 843-852.

17 A. Velyvis and L. E. Kay, J. Am. Chem. Soc., 2013, 135, 9259-9262.

18 D. Kurzbach, G. Kontaxis, N. Coudevylle and R. Konrat, in Intrinsically Disordered Proteins Studied by NMR Spectroscopy, ed. I. Felli and R. Pierattelli, Springer, 2015.

19 L. Geist, M. A. Henen, S. Haiderer, T. C. Schwarz, D. Kurzbach, A. Zawadzka-Kazimierczuk, S. Saxena, S. Zerko, W. Kozminski, D. Hinderberger and R. Konrat, Protein Sci., 2013, 22, 1196-1205.

20 D. Kurzbach, G. Platzer, T. C. Schwarz, M. A. Henen, R. Konrat and D. Hinderberger, Biochemistry, 2013, 52, 5167-5175.
21 G. Platzer, A. Schedlbauer, A. Chemelli, P. Ozdowy, N. Coudevylle, R. Auer, G. Kontaxis, M. Hartl, A. J. Miles, B. A. Wallace, O. Glatter, K. Bister and R. Konrat, Biochemistry, 2011, 50, 6113-6124.

22 J. Iwahara, C. Tang and G. M. Clore, J. Magn. Reson., 2007, 184, 185-195.

23 L. E. Kay and T. E. Bull, J. Magn. Reson., 1992, 99, 615-622.

24 R. Owenius, G. R. Eaton and S. S. Eaton, J. Magn. Reson., 2013, 236, 47-56.

25 I. V. Koptyug, S. H. Bossmann and N. J. Turro, J. Am. Chem. Soc., 1996, 118, 1435-1445.

26 G. Zwangenberg and P. J. Hore, Chem. Phys. Lett., 1993, 203, 65-74.

27 M. Carvetta, O. G. Johannessen and M. H. Levitt, Phys. Rev. Lett., 2004, 92, 153003.

28 R. Sarkar, P. Ahuja, P. R. Vasos and G. Bodenhausen, Phys. Rev. Lett., 2010, 104, 053001.

29 F. Delaglio, S. Grzesiek, G. W. Vuister, G. Zhu, J. Pfeifer and A. Bax, J. Biomol. NMR, 1995, 6, 277-293.

30 S. Stoll and A. Schweiger, J. Magn. Reson., 2006, 178, 42-55. 31 S. Stoll and A. Schweiger, Biol. Magn. Reson., 2007, 27, 299-321. 